\title{
Higher order and top mass effects in Higgs boson pair production beyond the Standard Model
}

\author{
Gerhard Buchalla \\ Ludwig-Maximilians-Universität München, Fakultät für Physik, Arnold Sommerfeld Center for \\ Theoretical Physics, 80333 München, Germany \\ E-mail: gerhard.buchalla@physik.uni-muenchen.de
}

\section{Matteo Capozi}

Max Planck Institute for Physics, Föhringer Ring 6, 80805 München, Germany

E-mail: mcapozi@mpp.mpg.de

\author{
Alejandro Celis \\ Ludwig-Maximilians-Universität München, Fakultät für Physik, Arnold Sommerfeld Center for \\ Theoretical Physics, 80333 München, Germany \\ E-mail: alejandro.celis@physik.uni-muenchen.de
}

\section{Gudrun Heinrich*}

Max Planck Institute for Physics, Föhringer Ring 6, 80805 München, Germany

E-mail: gudrun@mpp.mpg • de

\section{Ludovic Scyboz}

Max Planck Institute for Physics, Föhringer Ring 6, 80805 München, Germany

E-mail: scyboz@mpp.mpg • de

\begin{abstract}
We discuss the interplay between NLO QCD corrections and anomalous couplings in Higgs boson pair production via gluon fusion, within the framework of a non-linearly realised Effective Field Theory, described by the electroweak chiral Lagrangian. We study how the NLO corrections with full top quark mass dependence affect the total cross sections as well as distributions in the Higgs boson pair invariant mass. For a large part of the parameter space, significant and non-homogeneous K-factors arise.
\end{abstract}

Loops and Legs in Quantum Field Theory (LL2018)

29 April 2018 - 04 May 2018

St. Goar, Germany

${ }^{*}$ Speaker. 


\section{Introduction}

At the energy scales which colliders probe currently and in the nearer future, physics beyond the Standard Model (BSM) may manifest itself indirectly, for example via anomalous couplings in the Higgs sector. Some of the Higgs boson couplings, in particular the self-coupling, are still largely unconstrained and leave room for New Physics [1]. Assuming a New Physics scale $\Lambda$ in the $\mathrm{TeV}$ range or above, the BSM effects can be parametrised in a model-independent way in an Effective Field Theory (EFT) framework, where we can distinguish two main categories, often called "linear EFT" and "non-linear EFT". While the linear EFTs [2, 3], also known as "SMEFT" [4], are formulated as power series in the dimensionful parameter $1 / \Lambda$, the non-linear EFTs are organised by chiral dimensions and therefore the formalism is also called "Electroweak Chiral Lagrangian" (EWChL) framework. Prominent features of this approach are that the anomalous Higgs couplings are singled out systematically as the dominant New Physics effects in the electroweak sector, and that the Higgs field is an electroweak singlet. For more details we refer to Refs. [5, 6, 7] and references therein.

Higgs boson pair production in gluon fusion is the most promising process to measure the Higgs boson self-coupling and possibly other (effective) couplings involving more than one Higgs boson. In the Standard Model (SM), Higgs boson pair production has been calculated at leading order in Refs. [8,9]. As it is a loop-induced process, higher order calculations with full top quark mass dependence involve multi-scale two-loop integrals. Therefore, the NLO calculations until recently have been performed in the $m_{t} \rightarrow \infty$ limit [10], also called HTL or HEFT ("Higgs Effective Field Theory") ${ }^{1}$, and then rescaled by a factor $B_{F T} / B_{H E F T}$, where $B_{F T}$ denotes the leading order matrix element squared in the full theory. This procedure is called "Born-improved HEFT" in the

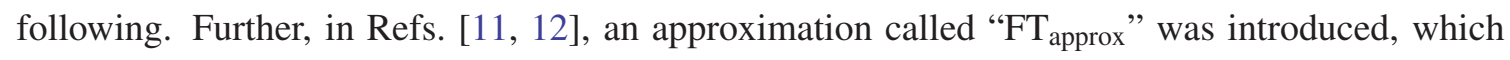
contains the full top quark mass dependence in the Born and real radiation parts, while the virtual part is calculated in the Born-improved HEFT approximation.

The full NLO corrections, including the top quark mass dependence also in the virtual twoloop amplitudes, have been calculated in Ref. [13], based on a numerical evaluation of the multiscale two-loop integrals with the program SECDEC [14, 15]. Phenomenological studies at $14 \mathrm{TeV}$ and $100 \mathrm{TeV}$, including variations of the Higgs boson self-coupling, have been presented in Ref. [16]. The full NLO calculation was supplemented by NLL transverse momentum resummation in Ref. [17]. It also has been matched to parton shower Monte Carlo programs [18, 19].

The NNLO QCD corrections in the heavy-top limit have been computed in Refs. [20, 21, 22, 23], and they have been supplemented by an expansion in $1 / m_{t}^{2}$ in Ref. [24] and by threshold resummation [25, 26]. In Ref. [27], top quark mass effects have been incorporated in the NNLO HEFT calculation, including the full NLO result and combining one-loop double-real corrections with full top mass dependence with suitably reweighted real-virtual and double-virtual contributions evaluated in the large- $m_{t}$ approximation. Very recently, threshold resummation on top of the latter result has been worked out in Ref. [28].

\footnotetext{
${ }^{1}$ Sometimes the electroweak chiral Lagrangian with a light Higgs boson is also referred to as Higgs Effective Field Theory (HEFT) in the literature. To avoid confusion, we will employ here the term electroweak chiral Lagrangian for the non-linear EFT of physics beyond the SM, and reserve the expression HEFT for the heavy-top limit.
} 
Within a non-linear EFT framework, higher order QCD corrections have been performed in the $m_{t} \rightarrow \infty$ limit. The NLO QCD corrections have been calculated in Ref. [29], including the case of CP-violating Higgs sectors [30]. The NNLO QCD corrections in the $m_{t} \rightarrow \infty$ limit including dimension 6 operators have been presented in Ref. [31]. These calculations found rather flat Kfactors, which however could be an artefact of the $m_{t} \rightarrow \infty$ limit.

Here we investigate whether this feature is preserved once the full top quark mass dependence is taken into account at NLO QCD, and quantify the effects of five operators that can lead to deviations from the SM in the Higgs sector.

\section{Setup of the calculation}

The terms in the effective Lagrangian relevant to our analysis are given by

$$
\mathscr{L} \supset-m_{t}\left(c_{t} \frac{h}{\mathrm{v}}+c_{t t} \frac{h^{2}}{\mathrm{v}^{2}}\right) \bar{t} t-c_{h h h} \frac{m_{h}^{2}}{2 \mathrm{v}} h^{3}+\frac{\alpha_{s}}{8 \pi}\left(c_{g g h} \frac{h}{\mathrm{v}}+c_{g g h h} \frac{h^{2}}{\mathrm{v}^{2}}\right) G_{\mu \nu}^{a} G^{a, \mu v} .
$$

To lowest order in the SM, $c_{t}=c_{h h h}=1$ and $c_{t t}=c_{g g h}=c_{g g h h}=0$. In principle, all couplings may have arbitrary values of $\mathscr{O}(1)$. The leading-order diagrams are shown in Fig. 1. Examples of

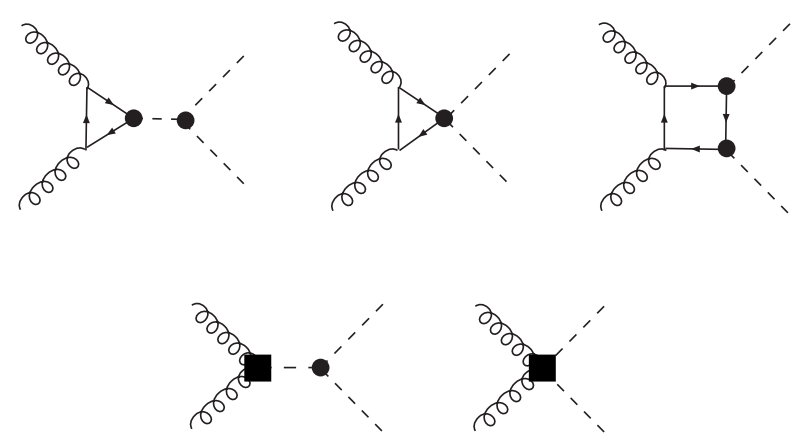

Figure 1: Higgs-pair production in gluon fusion at leading order in the chiral Lagrangian.

virtual NLO diagrams are shown in Fig. 2. For the two-loop part, we made use of the numerical results for the two-loop virtual diagrams in the SM $[13,16]$ by dividing them into two classes: diagrams containing the Higgs-boson self-coupling ("triangle-type"), and diagrams without ("boxtype"). The $t \bar{t} h h$ coupling generates new two-loop topologies, see e.g. the second line of Fig. 2. The results for these diagrams however can be obtained from the SM triangle-type diagrams by omitting the $s$-channel Higgs boson propagator and multiplying with $c_{t t} / c_{h h h}$. The other two-loop diagrams occurring in our calculation have the same topologies as in the SM and therefore can be obtained by rescaling of the couplings.

The real corrections consist of 5-point one-loop topologies with closed top quark loops as well as tree-level diagrams. Both classes of diagrams have been generated with GOSAM [32, 33] in combination with a model file in UFO format [34], derived from our effective Lagrangian using FEYnRULes [35]. The various building blocks are assembled in a $\mathrm{C}++$ program and integrated over the phase space using the CUBA library [36]. 

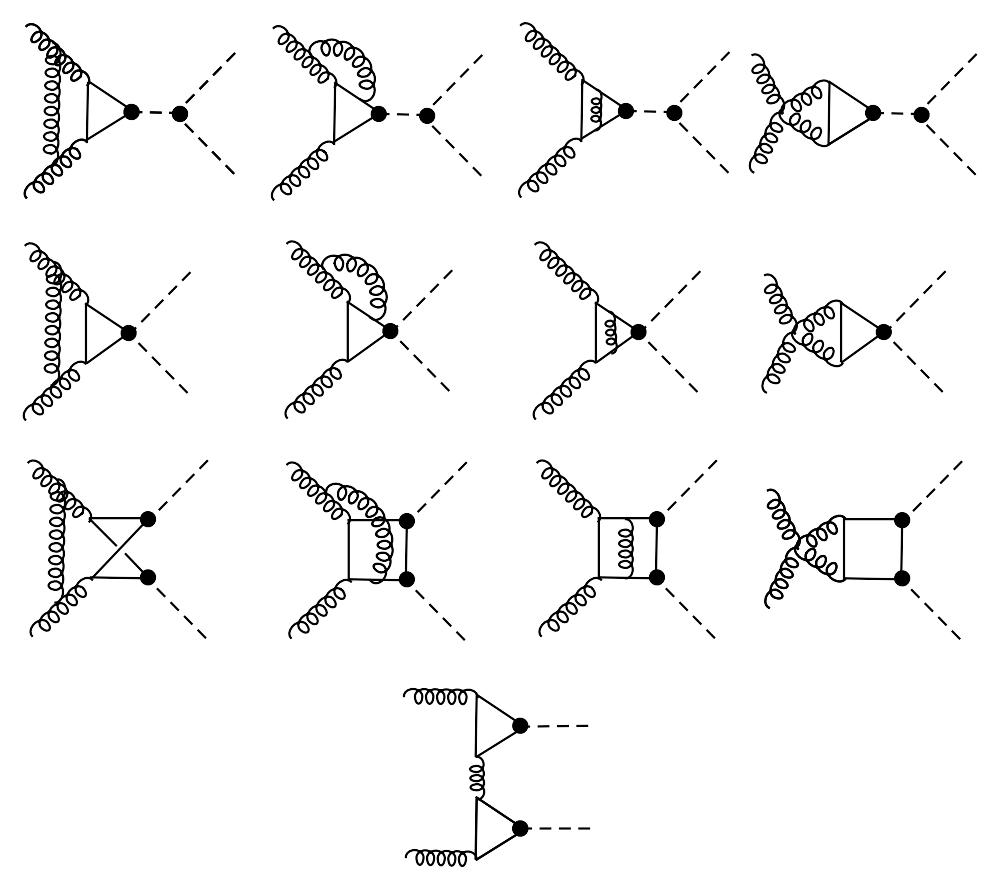

Figure 2: Higgs-pair production in gluon fusion at NLO: Examples for virtual two-loop diagrams at order $g_{s}^{4}$.

\section{Results}

All our results are for a centre-of-mass energy of $\sqrt{s}=14 \mathrm{TeV}$, computed using $m_{h}=125 \mathrm{GeV}$, $m_{t}=173 \mathrm{GeV}$ and the PDF4LHC15_nlo_100_pdfas [37] parton distribution functions. The widths of the top quark (and the Higgs boson) have been set to zero. Bottom quarks are treated as massless and therefore are not included in the fermion loops. The scale uncertainties are estimated by varying the factorisation scale $\mu_{F}$ and the renormalisation scale $\mu_{R}$ around the central scale $\mu_{0}=m_{h h} / 2$, using the envelope of a 7-point scale variation.

\subsection{Quantifying the NLO corrections}

The total cross section can be written in terms of the 15 coefficients $A_{1}, \ldots, A_{15}$, at $\mathrm{LO}[38,39]$ and in terms of 23 coefficients at NLO [5].

$$
\begin{aligned}
\sigma^{\mathrm{NLO}} / \sigma_{S M}^{\mathrm{NLO}}= & A_{1} c_{t}^{4}+A_{2} c_{t t}^{2}+A_{3} c_{t}^{2} c_{h h h}^{2}+A_{4} c_{g g h}^{2} c_{h h h}^{2}+A_{5} c_{g g h h}^{2}+A_{6} c_{t t} c_{t}^{2}+A_{7} c_{t}^{3} c_{h h h} \\
& +A_{8} c_{t t} c_{t} c_{h h h}+A_{9} c_{t t} c_{g g h} c_{h h h}+A_{10} c_{t t} c_{g g h}+A_{11} c_{t}^{2} c_{g g h} c_{h h h}+A_{12} c_{t}^{2} c_{g g h h} \\
& +A_{13} c_{t} c_{h h h}^{2} c_{g g h}+A_{14} c_{t} c_{h h h} c_{g g h h}+A_{15} c_{g g h} c_{h h h} c_{g g h h} \\
& +A_{16} c_{t}^{3} c_{g g h}+A_{17} c_{t} c_{t t} c_{g g h}+A_{18} c_{t} c_{g g h}^{2} c_{h h h}+A_{19} c_{t} c_{g g h} c_{g g h h} \\
& +A_{20} c_{t}^{2} c_{g g h}^{2}+A_{21} c_{t t} c_{g g h}^{2}+A_{22} c_{g g h}^{3} c_{h h h}+A_{23} c_{g g h}^{2} c_{g g h h} .
\end{aligned}
$$

Based on our results for $A_{1}, \ldots, A_{23}$, we produced heat maps for the ratio $\sigma / \sigma_{S M}$, varying two of the five parameters, while for the fixed parameters the SM values are used, along with $\sigma_{S M}^{\mathrm{LO}}=19.85 \mathrm{fb}$, 
$\sigma_{S M}^{\mathrm{NLO}}=32.95 \mathrm{fb}$. The couplings are varied in a range which seems reasonable when taking into account the current constraints on the Higgs coupling measurements [40], as well as recent limits on the di-Higgs production cross section [41, 42].

Fig. 3 shows the ratio to the SM total cross section both at LO and at NLO for variations of the triple Higgs coupling $c_{h h h}$ in combination with $c_{g g h}$ and $c_{t t}$. We observe that the deviations from the SM cross section as well as the effects of the NLO corrections can be substantial. Further we see a rapid variation of the cross section when changing $c_{t t}$ or $c_{h h h}$, while it is less sensitive to changes of $c_{g g h}$. In Fig. 4 we show the K-factors as a function of the five coupling parameters

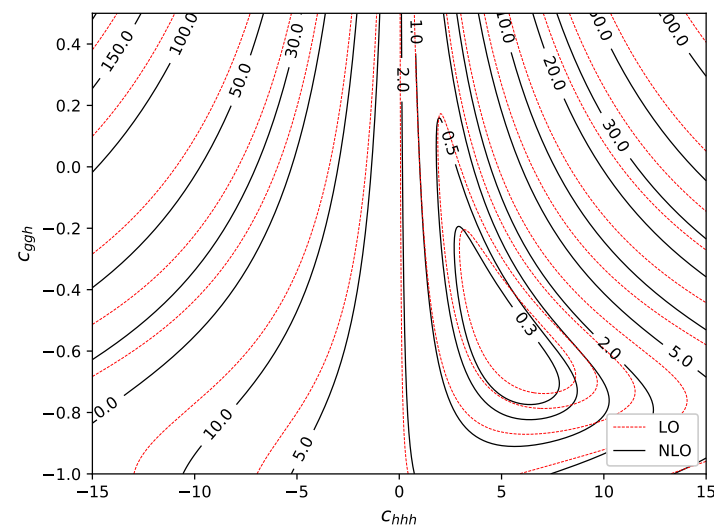

(a)

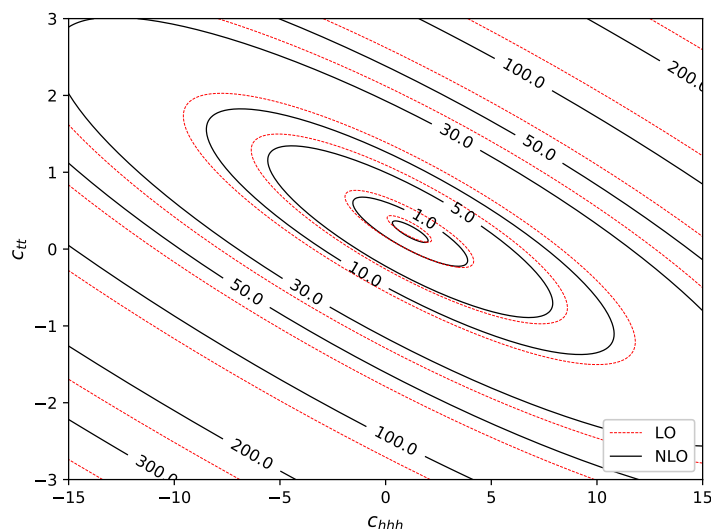

(b)

Figure 3: (a) $c_{g g h}$ versus $c_{h h h}$ and (b) $c_{t t}$ versus $c_{h h h}$.

(the fixed ones having SM values). It shows that the $\mathrm{K}$-factors show a rather strong dependence on these parameters, which was not the case in the $m_{t} \rightarrow \infty$ limit [29, 31].

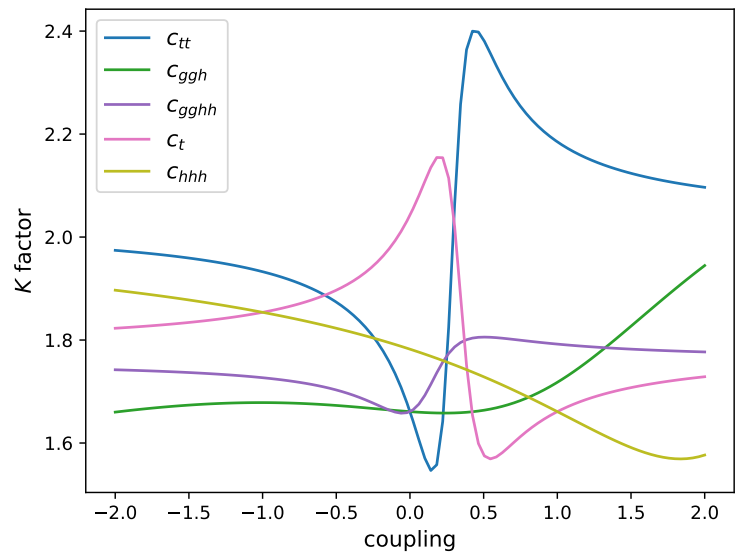

Figure 4: K-factors for the total NLO cross section as a function of the different couplings. 


\subsection{Total cross sections and distributions at benchmark points}

In Table 1 we show results for two benchmark points, labeled as benchmarks 6 and 9 in Ref. [39], where (in our conventions) the values for benchmark point 6 are $c_{h h h}=2.4, c_{t}=1, c_{t t}=$ $0, c_{g g h}=2 / 15, c_{g g h h}=1 / 15$, and the ones for benchmark point 9 are $c_{h h h}=1, c_{t}=1, c_{t t}=1, c_{g g h}=$ $-0.4, c_{g g h h}=-0.2$. The corresponding total cross sections are shown in Table 1 . Results for 10 more benchmark points can be found in Ref. [5].

\begin{tabular}{|c|c|c|c|c|c|}
\hline Benchmark & $\sigma_{N L O}[\mathrm{fb}]$ & K-factor & scale uncert. [\%] & stat. uncert. [\%] & $\frac{\sigma_{N L O}}{\sigma_{N L O S M}}$ \\
\hline$B_{6}$ & 24.69 & 1.89 & ${ }_{-11}^{+2}$ & 2.1 & 0.7495 \\
\hline$B_{9}$ & 146.00 & 2.30 & ${ }_{-16}^{+22}$ & 0.31 & 4.431 \\
\hline$S M$ & 32.95 & 1.66 & ${ }_{-13}^{+14}$ & 0.1 & 1 \\
\hline
\end{tabular}

Table 1: Total cross sections at NLO (second column) including the K-factor (third column), scale uncertainties (4th column) and statistical uncertainties (5th column) and the ratio to the SM total NLO cross section (6th column).

Now we consider the Higgs boson pair invariant mass distribution for the two benchmark points. The full NLO result is shown in red, the two approximations "Born-improved NLO HEFT" (purple) and $\mathrm{FT}_{\text {approx }}$ (green) are also shown. The leading order BSM results are shown in yellow, the SM results are shown in blue (NLO) and black (LO). The lower ratio plot shows the ratio of the two approximate results to the full NLO result. The upper ratio plot shows the differential BSM K-factor, i.e. $\mathrm{NLO}_{\mathrm{BSM}} / \mathrm{LO}_{\mathrm{BSM}}$, both evaluated with the same PDFs.

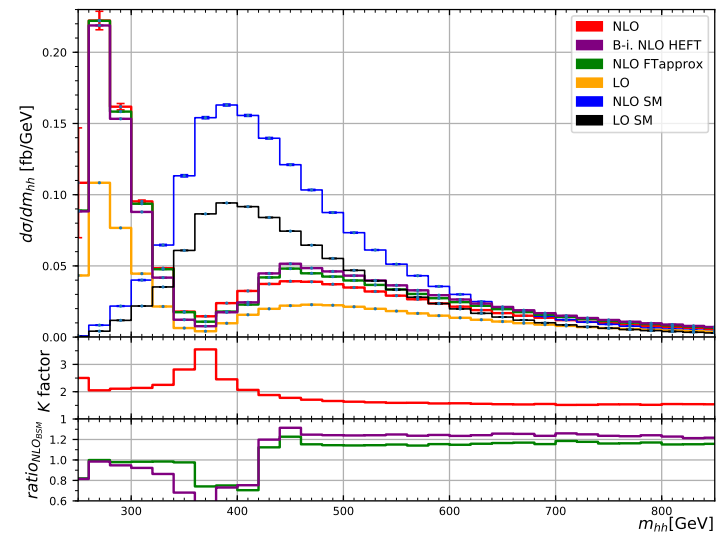

(a)

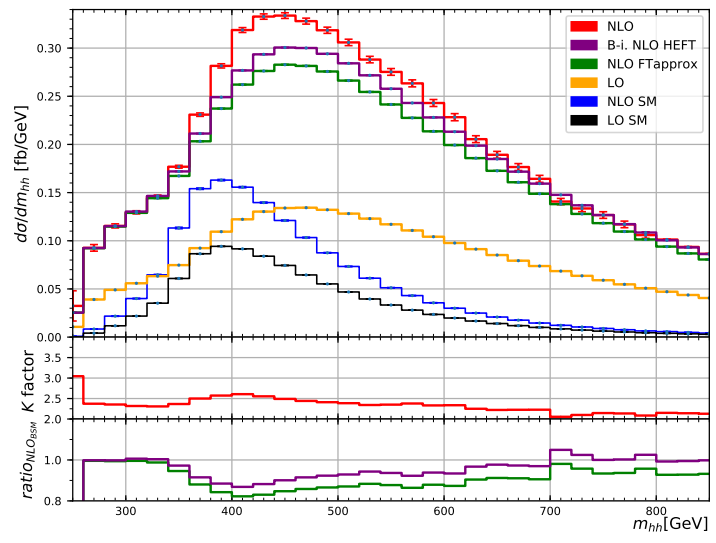

(b)

Figure 5: Higgs boson pair invariant mass distribution for (a): Benchmark point $6, c_{h h h}=2.4, c_{t}=$ $1, c_{t t}=0, c_{g g h}=2 / 15, c_{g g h h}=1 / 15$, and (b) benchmark point $9, c_{h h h}=1, c_{t}=1, c_{t t}=1, c_{g g h}=$ $-0.4, c_{g g h h}=-0.2$.

Benchmark point 6 shows a dip where the SM $m_{h h}$ distribution has a peak, related to the fact that the LO HEFT amplitude exactly vanishes at $m_{h h}=429 \mathrm{GeV}$. In addition it shows a large enhancement of the low $m_{h h}$ region due to the value $c_{h h h}=2.4$. Note that this value for $c_{h h h}$ is very 
close to the point where the total cross section as a function of $c_{h h h}$ goes through a minimum if all other couplings are kept SM-like. Even though the values for $c_{t}$ and $c_{t t}$ are the same as in the SM, the shape of the $m_{h h}$ distribution for benchmark 6 is very different from the SM one and therefore would be a very characteristic sign of anomalous couplings.

For benchmark point 9 , the values for $c_{h h h}$ and $c_{t}$ are as in the SM, however the total cross section is more than four times larger. The enhancement is particularly pronounced in the tails of the distributions, which can be attributed mainly to the rather large absolute values of $c_{g g h}$ and $c_{g g h h}$, in combination with a non-zero value of $c_{t t}$.

\section{Conclusions}

We have presented a calculation of the NLO QCD corrections with full $m_{t}$ dependence to Higgs boson pair production within the framework of a non-linearly realised Effective Field Theory (Electroweak Chiral Lagrangian) in the Higgs sector. This framework, applied to $g g \rightarrow h h$, allows us to focus on five anomalous Higgs boson couplings, $c_{h h h}, c_{t}, c_{t t}, c_{g g h}$ and $c_{g g h h}$.

Our calculation is based on a numerical evaluation of two-loop multi-scale integrals which so far are not accessible analytically. In particular, the results for two-loop integrals involving an effective $h h t \bar{t}$ contact interaction, parametrised by $c_{t t}$, allowed us to study for the first time the effect of such an anomalous coupling including full NLO QCD corrections. We found that the cross sections are quite sensitive to variations of $c_{t t}$, while variations of the Higgs-gluon effective interactions $c_{g g h}$ and $c_{g g h h}$ have a weaker effect on the total cross sections. For the considered benchmark points, the NLO K-factors are of the order of two for the total cross sections, however they can vary by up to $\pm 20 \%$ as the anomalous couplings are varied. The differential K-factors for the $m_{h h}$ distribution show even stronger variations, in particular around the threshold region $m_{h h} \sim 2 m_{t}$. This emphasises the importance of including the NLO QCD corrections with full top quark mass dependence in studies of anomalous couplings in the Higgs sector.

\section{References}

[1] G. Brooijmans et al., Les Houches 2017: Physics at TeV Colliders New Physics Working Group Report, in 10th Les Houches Workshop on Physics at TeV Colliders (PhysTeV 2017) Les Houches, France, June 5-23, 2017, 2018. 1803.10379.

[2] W. Buchmüller and D. Wyler, Effective Lagrangian Analysis of New Interactions and Flavor Conservation, Nucl. Phys. B268 (1986) 621-653.

[3] B. Grzadkowski, M. Iskrzynski, M. Misiak, and J. Rosiek, Dimension-Six Terms in the Standard Model Lagrangian, JHEP 10 (2010) 085, [1 008 . 4884].

[4] L. Berthier and M. Trott, Towards consistent Electroweak Precision Data constraints in the SMEFT, JHEP 05 (2015) 024, [1502 . 02570].

[5] G. Buchalla, M. Capozi, A. Celis, G. Heinrich, and L. Scyboz, Higgs boson pair production in non-linear Effective Field Theory with full $m_{t}$-dependence at NLO QCD, 1806.05162.

[6] R. Alonso, M. B. Gavela, L. Merlo, S. Rigolin, and J. Yepes, The Effective Chiral Lagrangian for a Light Dynamical "Higgs Particle", Phys. Lett. B722 (2013) 330-335, [1212 . 330 5]. [Erratum: Phys. Lett.B726,926(2013)]. 
[7] G. Buchalla, O. Cata, and C. Krause, Complete Electroweak Chiral Lagrangian with a Light Higgs at NLO, Nucl. Phys. B880 (2014) 552-573, [1307. 5017 ]. [Erratum: Nucl. Phys.B913,475(2016)].

[8] O. J. P. Eboli, G. C. Marques, S. F. Novaes, and A. A. Natale, Twin Higgs Boson Production, Phys. Lett. B197 (1987) 269.

[9] E. W. N. Glover and J. J. van der Bij, Higgs Boson Pair Production via Gluon Fusion, Nucl. Phys. B309 (1988) 282.

[10] S. Dawson, S. Dittmaier, and M. Spira, Neutral Higgs boson pair production at hadron colliders: QCD corrections, Phys. Rev. D58 (1998) 115012, [hep-ph/9805244].

[11] R. Frederix, S. Frixione, V. Hirschi, F. Maltoni, O. Mattelaer, P. Torrielli, E. Vryonidou, and M. Zaro, Higgs pair production at the LHC with NLO and parton-shower effects, Phys. Lett. $\mathrm{B732}$ (2014) 142-149, [1401.7340].

[12] F. Maltoni, E. Vryonidou, and M. Zaro, Top-quark mass effects in double and triple Higgs production in gluon-gluon fusion at NLO, JHEP 11 (2014) 079, [1 408 . 6542].

[13] S. Borowka, N. Greiner, G. Heinrich, S. Jones, M. Kerner, J. Schlenk, U. Schubert, and T. Zirke, Higgs Boson Pair Production in Gluon Fusion at Next-to-Leading Order with Full Top-Quark Mass Dependence, Phys. Rev. Lett. 117 (2016), no. 1 012001, erratum ibid 079901, [1604.06447].

[14] S. Borowka, G. Heinrich, S. P. Jones, M. Kerner, J. Schlenk, and T. Zirke, SecDec-3.0: numerical evaluation of multi-scale integrals beyond one loop, Comput. Phys. Commun. 196 (2015) 470-491, [1502.06595].

[15] S. Borowka, G. Heinrich, S. Jahn, S. P. Jones, M. Kerner, J. Schlenk, and T. Zirke, pySecDec: $a$ toolbox for the numerical evaluation of multi-scale integrals, Comput. Phys. Commun. 222 (2018) 313-326, [1703.09692].

[16] S. Borowka, N. Greiner, G. Heinrich, S. P. Jones, M. Kerner, J. Schlenk, and T. Zirke, Full top quark mass dependence in Higgs boson pair production at NLO, JHEP 10 (2016) 107, [1608 04798 ].

[17] G. Ferrera and J. Pires, Transverse-momentum resummation for Higgs boson pair production at the LHC with top-quark mass effects, JHEP 02 (2017) 139, [1609. 01691 ].

[18] G. Heinrich, S. P. Jones, M. Kerner, G. Luisoni, and E. Vryonidou, NLO predictions for Higgs boson pair production with full top quark mass dependence matched to parton showers, JHEP 08 (2017) 088, [1703.09252].

[19] S. Jones and S. Kuttimalai, Parton Shower and NLO-Matching uncertainties in Higgs Boson Pair Production, JHEP 02 (2018) 176, [1711.03319].

[20] D. de Florian and J. Mazzitelli, Two-loop virtual corrections to Higgs pair production, Phys. Lett. B724 (2013) 306-309, [1305.5206].

[21] D. de Florian and J. Mazzitelli, Higgs Boson Pair Production at Next-to-Next-to-Leading Order in QCD, Phys. Rev. Lett. 111 (2013) 201801, [1309. 6594].

[22] J. Grigo, K. Melnikov, and M. Steinhauser, Virtual corrections to Higgs boson pair production in the large top quark mass limit, Nucl. Phys. $\mathbf{B 8 8 8}$ (2014) 17-29, [1408 .2422].

[23] D. de Florian, M. Grazzini, C. Hanga, S. Kallweit, J. M. Lindert, P. Maierhöfer, J. Mazzitelli, and D. Rathlev, Differential Higgs Boson Pair Production at Next-to-Next-to-Leading Order in QCD, JHEP 09 (2016) 151, [1606.09519]. 
[24] J. Grigo, J. Hoff, and M. Steinhauser, Higgs boson pair production: top quark mass effects at NLO and NNLO, Nucl. Phys. B900 (2015) 412, [1508.00909].

[25] D. Y. Shao, C. S. Li, H. T. Li, and J. Wang, Threshold resummation effects in Higgs boson pair production at the LHC, JHEP 07 (2013) 169, [1301.1245].

[26] D. de Florian and J. Mazzitelli, Higgs pair production at next-to-next-to-leading logarithmic accuracy at the LHC, JHEP 09 (2015) 053, [1505. 07122].

[27] M. Grazzini, G. Heinrich, S. Jones, S. Kallweit, M. Kerner, J. M. Lindert, and J. Mazzitelli, Higgs boson pair production at NNLO with top quark mass effects, JHEP 05 (2018) 059, [1 803.02463 ].

[28] D. de Florian and J. Mazzitelli, Soft gluon resummation for Higgs boson pair production including finite Mt effects, 1807.03704.

[29] R. Gröber, M. Mühlleitner, M. Spira, and J. Streicher, NLO QCD Corrections to Higgs Pair Production including Dimension-6 Operators, JHEP 09 (2015) 092, [1 504.06577$].$

[30] R. Gröber, M. Mühlleitner, and M. Spira, Higgs Pair Production at NLO QCD for CP-violating Higgs Sectors, Nucl. Phys. B925 (2017) 1-27, [1705. 05314$].$

[31] D. de Florian, I. Fabre, and J. Mazzitelli, Higgs boson pair production at NNLO in QCD including dimension 6 operators, JHEP 10 (2017) 215, [1704 . 05700].

[32] G. Cullen, N. Greiner, G. Heinrich, G. Luisoni, P. Mastrolia, G. Ossola, T. Reiter, and F. Tramontano, Automated One-Loop Calculations with GoSam, Eur. Phys. J. C72 (2012) 1889, [1111. 2034 ].

[33] G. Cullen et al., GoSam-2.0: a tool for automated one-loop calculations within the Standard Model and beyond, Eur. Phys. J. C74 (2014), no. 8 3001, [1404.7096].

[34] C. Degrande, C. Duhr, B. Fuks, D. Grellscheid, O. Mattelaer, and T. Reiter, UFO - The Universal FeynRules Output, Comput. Phys. Commun. 183 (2012) 1201-1214, [1108. 2040 ].

[35] A. Alloul, N. D. Christensen, C. Degrande, C. Duhr, and B. Fuks, FeynRules 2.0 - A complete toolbox for tree-level phenomenology, Comput. Phys. Commun. 185 (2014) 2250-2300, [1310.1921].

[36] T. Hahn, CUBA: A Library for multidimensional numerical integration, Comput. Phys. Commun. 168 (2005) 78-95, [hep-ph/0404043].

[37] J. Butterworth et al., PDF4LHC recommendations for LHC Run II, J. Phys. G43 (2016) 023001, [1510.03865].

[38] A. Azatov, R. Contino, G. Panico, and M. Son, Effective field theory analysis of double Higgs boson production via gluon fusion, Phys. Rev. D92 (2015), no. 3 035001, [1502.00539].

[39] A. Carvalho, M. Dall'Osso, T. Dorigo, F. Goertz, C. A. Gottardo, and M. Tosi, Higgs Pair Production: Choosing Benchmarks With Cluster Analysis, JHEP 04 (2016) 126, [1507. 02245$].$

[40] ATLAS, CMS Collaboration, G. Aad et al., Measurements of the Higgs boson production and decay rates and constraints on its couplings from a combined ATLAS and CMS analysis of the LHC pp collision data at $\sqrt{s}=7$ and 8 TeV, JHEP 08 (2016) 045, [1606. 022 66].

[41] CMS Collaboration, A. M. Sirunyan et al., Search for Higgs boson pair production in the $\gamma \gamma \mathrm{b} \overline{\mathrm{b}}$ final state in pp collisions at $\sqrt{s}=13 \mathrm{TeV}, 1806.00408$.

[42] ATLAS Collaboration, M. Aaboud et al., Search for Higgs boson pair production in the $\gamma \gamma b \bar{b}$ final state with $13 \mathrm{TeV}$ pp collision data collected by the ATLAS experiment, 1807.04873. 